\title{
Development of Optimization Methodology for Short Range Air-to-Air Missile
}

Romie Oktovianus Bura ${ }^{1}$, Yudki Utama ${ }^{2}$, and Muhammad Farraz ${ }^{3}$

\begin{abstract}
This paper illustrates the development and the use of conceptual sizing tools integrated with optimization tools. The conceptual sizing tool is based on empirical and simple analytical calculation that simple and robust enough for wide range of inputs. The genetic algorithm optimization method then used to explore the global optimum for given variables. System response analysis is conducted to gain insight about the sensitivity of objective function because of design variables.
\end{abstract}

Keywords - Conceptual Design, Missile, Optimization, System Response Analysis, Evolutionary Algorithm.

\section{INTRODUCTION}

$\mathbf{M}$ ISSILE technology is an important part of achieving independent ability in developing weapon system and domestic defense industry. This is also important for Indonesia, which has participated in the development of fighter aircraft together with South Korea. Hence, air-to-air missile (as seen in Figure 1) development is a necessary parallel effort to arm the fighter. The missile development is a long process, starting from conceptual design to production and manufacturing. One of the most important phases in the design process is the conceptual phase of design, in which the initial shape of the rocket to be made is determined.

One of the methods already developed in conceptual rocket design is the design method by Fleeman [1]. In order to generate better design, an optimization method is applied on Fleeman method, starting from the missile aerodynamics, propulsion, trajectory, and finally the structure.

\section{METHODOLOGY}

The method used in this research is numerical method that is using equation of empiric equation and work flow on Fleeman method [1], by adding optimization method. A code for analysis and optimization is developed the conceptual design process starts from the definition of mission requirement and then selects the design baseline for short range air to air missile. Python programming language [2] is used in the code because the open source nature and its simplicity. The conceptual design is initiated in the aerodynamics, propulsion and structure parts by utilizing the optimization method.

The addition of optimization method to this Fleeman method will result in faster processing time and better design than by using Fleeman method alone. The optimization method applied is Genetic Algorithm. Genetic Algorithm works so that optimizations get the global optimum. In this initial work, the tools developed is limited to short range airto-air missile with focus on the aerodynamics, propulsion, trajectory, and structure conceptual design.

\section{A. Conceptual Design and Sizing Methods}

Fleeman method developed in this work is based on Tactical Missile Design (TMD) spreadsheet [3]. Tactical Missile Design (TMD) spreadsheet is a conceptual sizing tool for the design of tactical missile that includes analyses for many disciplines. The spreadsheet disciplines are aerodynamics, propulsion, trajectory, structure, warhead, radar, and dynamics. The spreadsheet calculation can be divided by two major calculation. The first calculations are an intercoupled calculation built from aerodynamics, propulsion, and trajectory calculations as shown in Fig. 1. Second vehicle calculations include structure, warhead, radar, and dynamics. However, because of the scope in this work mentioned before, the second calculation just include structure calculation. The primary calculation is not intrinsically connected with the second calculations. Outputs from the second calculation is not become inputs for primary calculation, but vice versa.

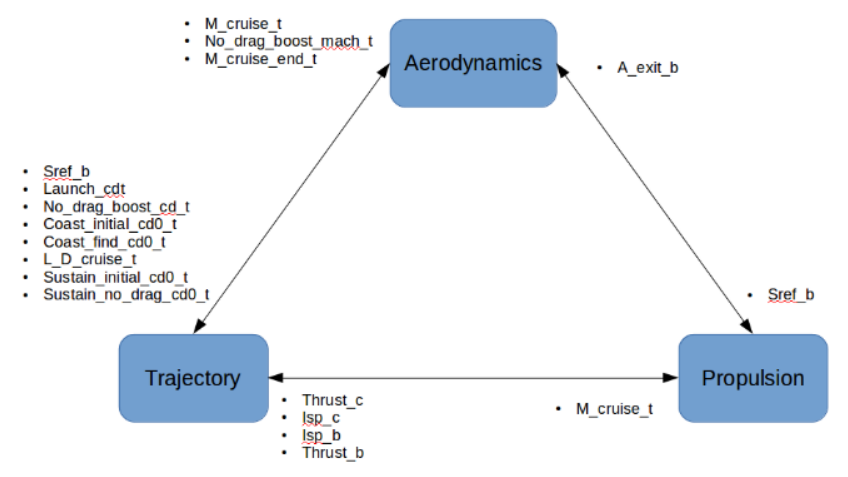

Fig. 1. Intercoupled primary range calculation.

\section{1) Primary Range/Flyout Calculation Coupling}

Aerodynamics

The aerodynamics discipline calculation is based on Sparrow Medium-Range Air-to-Air Missile (MRAAM). 


\section{Propulsion}

For propulsion calculation, according to the limitation mentioned before, the developed code operates with just one type of engine that is solid propellant. Solid propellant based propulsion is generally used for short range air-to-air missile.

\section{$\underline{\text { Trajectory }}$}

Trajectory calculation done with assumption that the missile flies a constant flight path angle. By assuming a constant flight angle, trajectory calculation can be built with simple one degree of freedom model.

\section{2) Secondary Vehicle Calculation}

\section{Structure}

As mentioned before, there is no feedback from the structure calculation to primary range calculation. From available material properties, expected loads on missile, and the maximum Mach number input, structure module produces structure sensitive estimation such as required motor dimensions and weight and the skin temperature of the missile.

\section{3) Convergence criteria}

One advantage to translate the TMD spreadsheet into a Python code is that some iterative process that could be tedious to be done in spreadsheet can be done easily. In this code, the iteration process is done whenever the iteration has reach the maximum iteration or the residual root mean square (rms) value from initial and calculated values of booster exit area, cruise Mach number, end Mach number from no drag equation, and end cruise Mach number is below rms criteria. Fig. 2. shows all residual rms mentioned before from an example case. In this case, the rms criteria default is 1e-5. As shown, the iteration stops at $4^{\text {th }}$ when residual $\mathrm{rms}$ reach $\mathrm{rms}$ criteria.

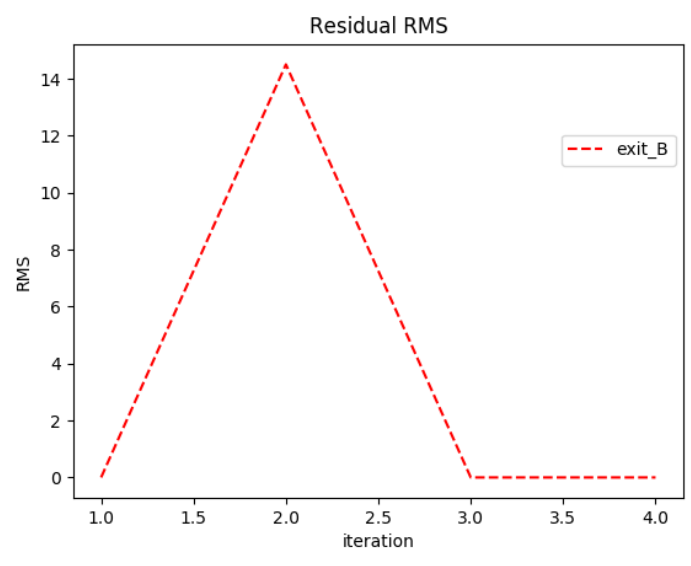

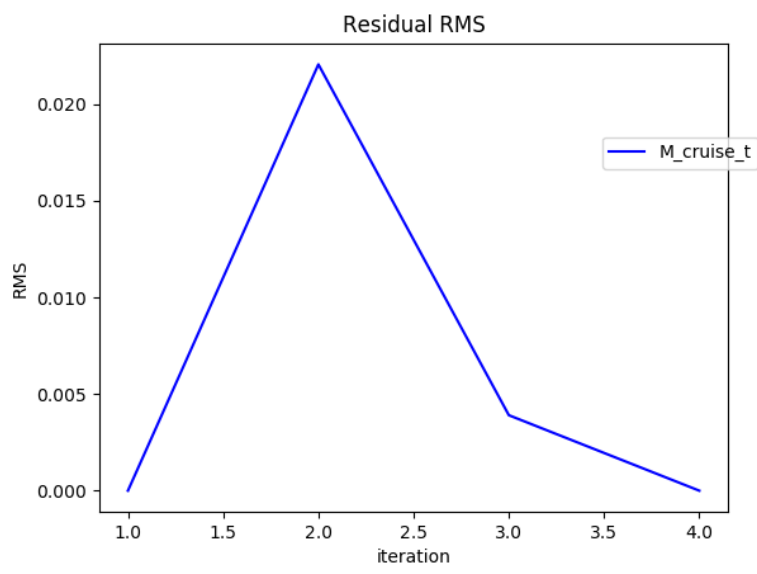
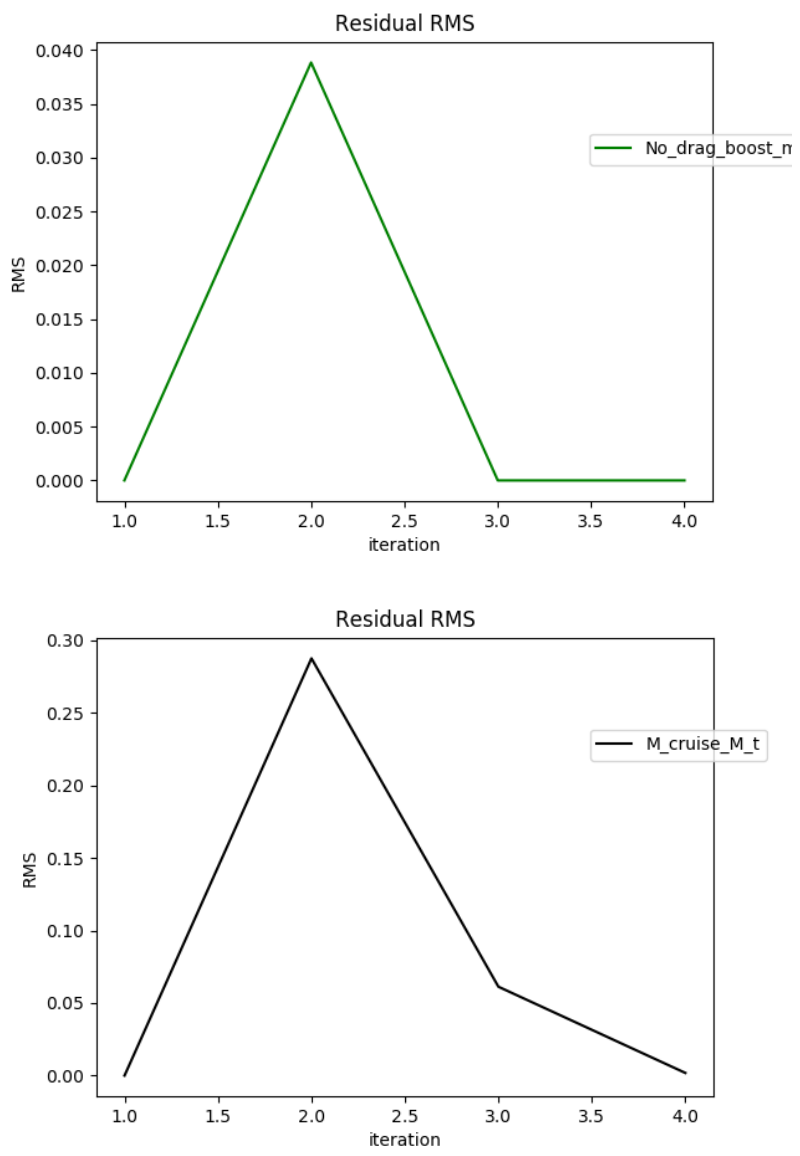

Fig. 2. Residual rms iteration history.

\section{B. Optimization Methods}

The optimization problem in this work refer to previous work by Frits as follows:

1. objective min flight time (Total_time_t))

2. constraints:

1. turn radius $<4000$ feet $=1219.2 \mathrm{~m}$ 


\section{2. flight range $>=10 \mathrm{nmi}=18520 \mathrm{~m}$ (Total_range_t $)$}

3. missile weight $\left.=500 \mathrm{lbm}=226,796 \mathrm{~kg}\left(W \_l a u n c h \_t\right)\right)$

3. variables:

1. launch weight (W_launch_t)

2. diameter (a2)

3. nose length ( $L N)$

4. wing area $(S W)$

5.expansion ratio $\left(e_{-} b, e_{-} c\right)$

6. boost chamber pressure $\left(P c_{-} b\right)$

7. sustain chamber pressure $\left.\left(P c_{-} c\right)\right)$

4. Optimization methods (GA)

\section{RESULTS}

A system response analysis was conducted to study how the objective function response with variation of design variables. Results of system response analysis is shown in Fig.3.
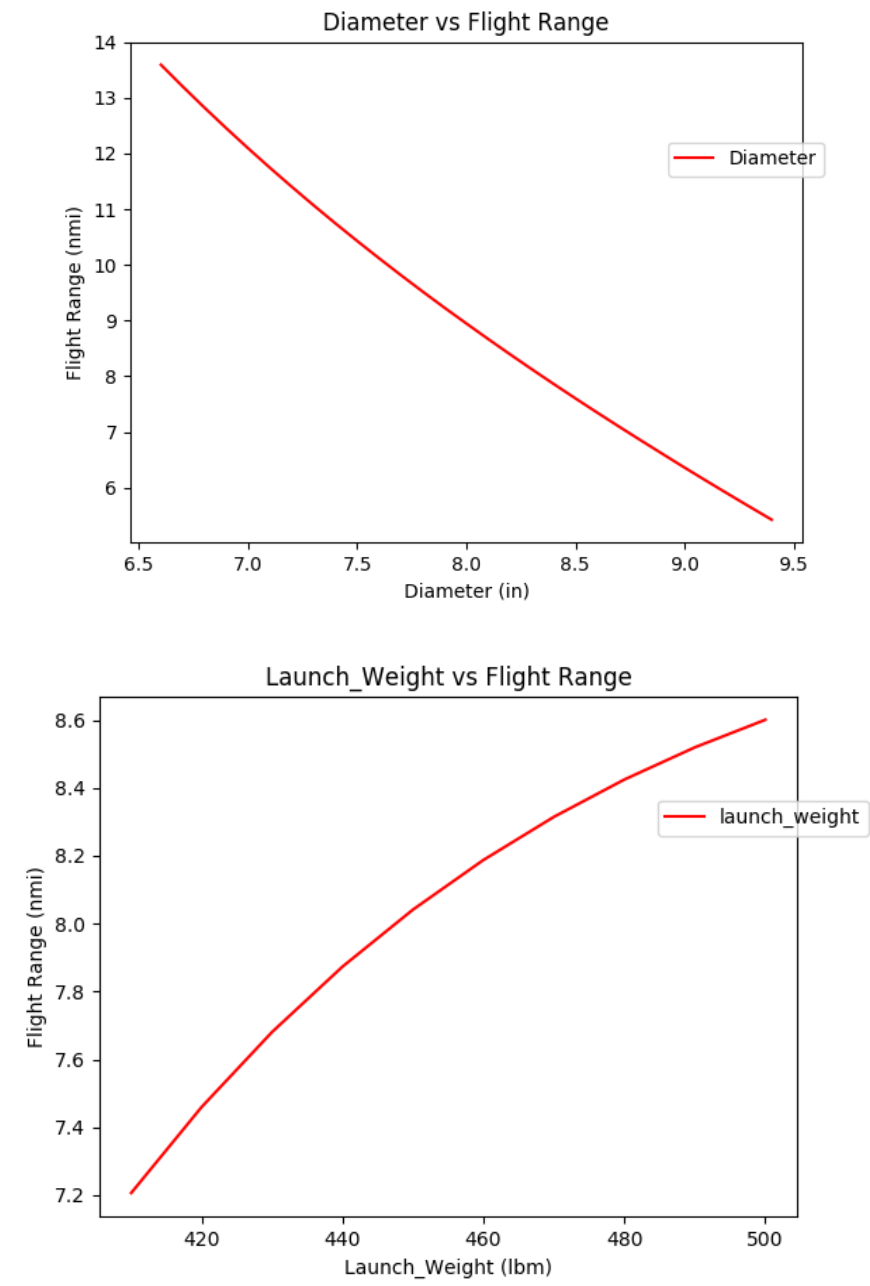
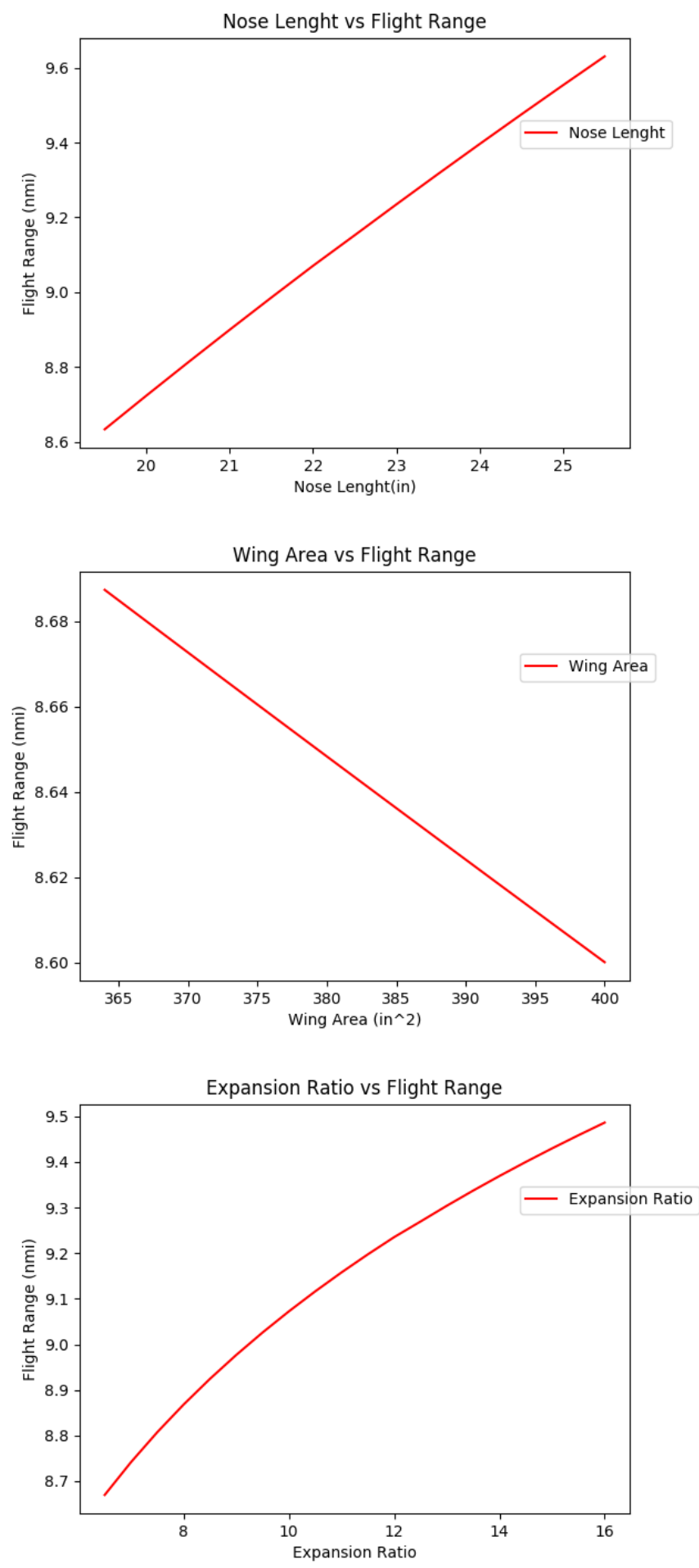

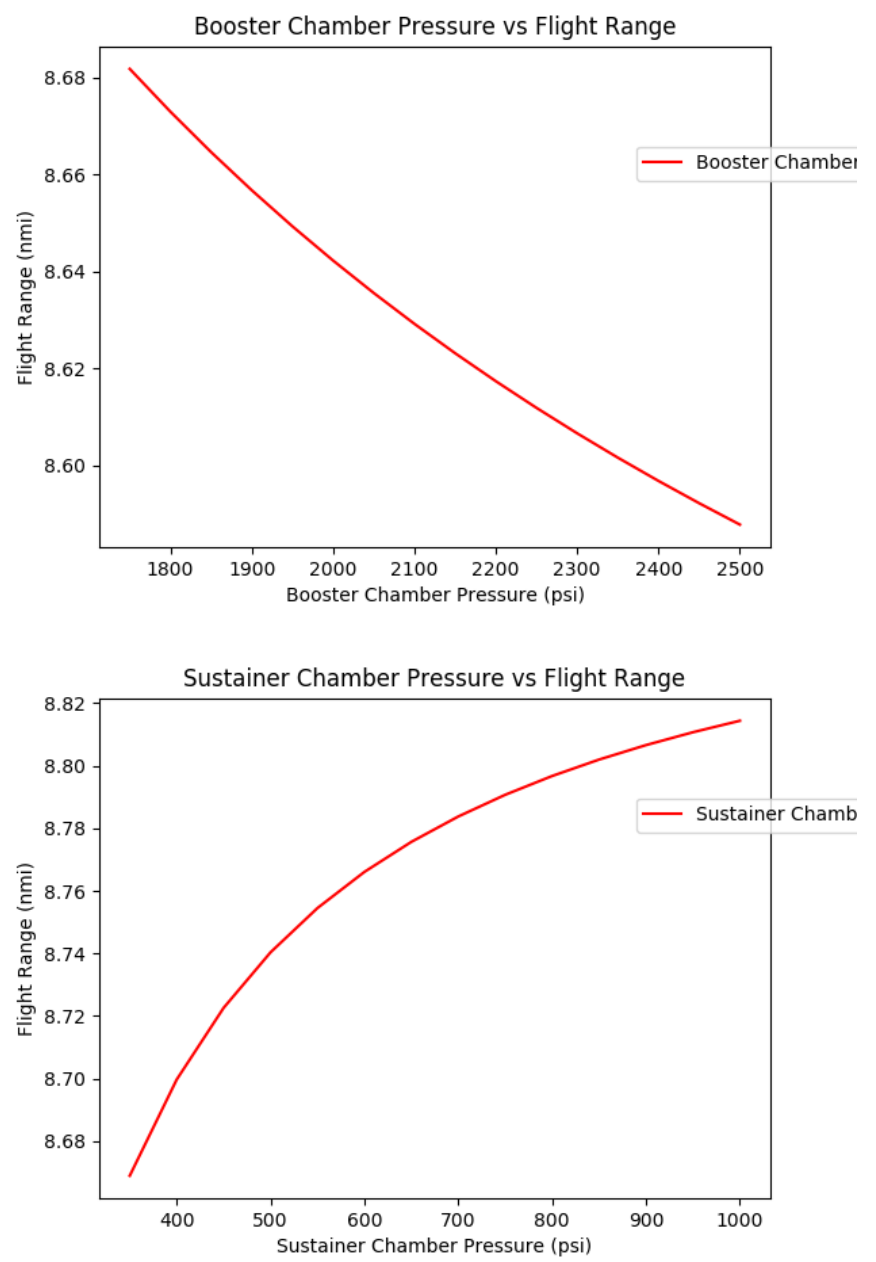

Fig. 3. System response analysis results

\section{CONCLUSION}

In this work, a Python code for primary range/flyout calculation was made. Genetic algorithm optimization was implemented in the code. Due to lack of missile databases for validation this work just conducted program verification with TMD Spreadsheet. Future works includes technology readiness consideration, dynamics and other modules integrating, new database generation, and development of all discipline prediction methods. To be able to implement technology readiness consideration, development of intercoupled primary and secondary calculation is mandatory, for example, calculation of the optimum aerodynamics, propulsion, and trajectory characteristic based on available material technology. Finally, this work will explore more on the optimization methods.

\section{REFERENCES}

[1] Fleeman, Eugene L. Tactical missile design. American Institute of Aeronautics and Astronautics, 2006.

[2] Frits, Andrew P., Eugene L. Fleeman, and Dimitri N. Mavris. "Use of a Conceptual Sizing Tool for Conceptual Design of Tactical Missiles (U)." .(2002).

[3] Van Rossum, Guido, and Fred L. Drake. Python 3: Reference Manual. SohoBooks, 2009. 